\title{
Phospholipid Adsorption Polymeric Materials for Detection of Xylazine and Metabolite in Blood and Urine
}

\author{
Xue Gao, ${ }^{1,2}$ Hao Guo, ${ }^{3}$ and Chaokang $\mathrm{Gu}^{4}$ \\ ${ }^{1}$ School of Environmental and Biological Engineering, Chongqing Technology and Business University, Chongqing 400067, China \\ ${ }^{2}$ Chongqing Key Lab of Catalysis \& Functional Organic Molecules, Chongqing Technology and Business University, \\ Chongqing 400067, China \\ ${ }^{3}$ Chongqing Institute of Forensic Science, Chongqing 400021, China \\ ${ }^{4}$ Reel Solar Power Inc., San Jose, CA 95131, USA
}

Correspondence should be addressed to Hao Guo; hao.guo1982@hotmail.com

Received 3 July 2016; Accepted 16 August 2016

Academic Editor: Yiwen Li

Copyright (c) 2016 Xue Gao et al. This is an open access article distributed under the Creative Commons Attribution License, which permits unrestricted use, distribution, and reproduction in any medium, provided the original work is properly cited.

Polymers have been used in different areas. Recently, polymeric material is favored in analytical area due to its high performance and high consistency, which was used in sample pretreatment in this study. Xylazine poisoning is often seen in body fluid samples obtained from various accidents or suicides. However, the content of xylazine is difficult to detect precisely due to matrix effect in testing practices. In this paper, a method application for phospholipid adsorption polymeric materials to determine xylazine in blood and urine samples was proposed, developed, and validated. Compared with existing method, this method using polymeric pretreatment has a wider linear range of $2.0-2000.0 \mathrm{ng} / \mathrm{mL}$ for xylazine and its metabolite 2,6-dimethylaniline in both blood and urine and lower detection limits of $0.3 \mathrm{ng} / \mathrm{mL}$ for 2,6-dimethylaniline and xylazine in blood and $0.2 \mathrm{ng} / \mathrm{mL}$ for 2,6-dimethylaniline and xylazine in urine. Therefore, this method is suggested to be applied in testing practices by academic groups and commercial organizations.

\section{Introduction}

The variety of polymers gives different research area different opportunities to achieve different functions. In the recent decades, natural polymers have been modified through grafting to conquer their born defects, including the poor solubility and poor mechanical properties [1-4]. Meanwhile, synthetic polymers have been engineered so much to achieve new functions, such as long-term antimicrobial polymers [5]. More importantly, polymers have been regarded as the most important component in composite material towards stronger and smarter materials [6-9] and more precise and more sensitive extraction materials $[10,11]$. Especially in the analytical field, polymers play an important role in separation and purification when dealing with novel materials [12,13] and complicated biosamples [14]. Blood is a complex sample that contains plasma, proteins, and other trace interferents that can often cause matrix effect when using electrospray ionization (ESI) technology. Therefore, the blood sample preparation is a very important step in the analysis process. In recent years, several new methods have been developed and applied to blood sample preparation, for example, protein and phospholipid removal plate; it has been used for the targeted removal of phospholipids from blood samples. The protocol can remove interferents in the blood as much as possible, especially proteins and phospholipids, resulting in lowered matrix effect and enhanced recovery. The operation of the protocol is simple and convenient, which only needs to conduct the protein precipitation inside the plate and then push samples to flow through the absorber. The resultant filtrate can be used directly in LC-MS detection.

Xylazine has been suggested to be used on various kinds of animals, including dogs, cats, horses, and deer, for antianxiety, sedative, and analgetic purposes [2]. The chemical structure of xylazine resembles that of phenothiazines, tricyclic antidepressants, and clonidine. Xylazine is a highly 


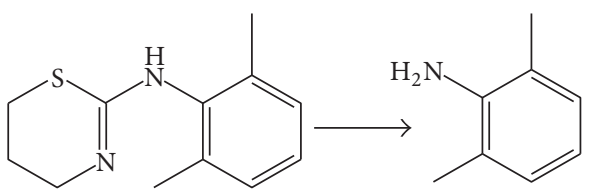

FIGURE 1: The structures of xylazine and DMA.

effective $\alpha$-2 adrenergic stimulant, which can stimulate $\alpha$ 2 receptors at the central nervous system. When those $\alpha-2$ receptors are stimulated, the central nervous system reduces the release of norepinephrine and dopamine, resulting in the effects of tranquilizer, painkiller, and muscle relaxant. Xylazine can also affect the cholinergic neuronal system, $\alpha$ 1 adrenergic receptor, and histamine system [15]. It has been reported that xylazine has been abused for its anesthetic effect either purposely or accidentally in intoxication and criminal cases $[16,17]$. Human bodies will show symptoms such as depressed central nervous system, depressed respiratory system, bradycardia, or low blood pressure when intoxicated with xylazine. The main xylazine biotransformation pathway is most likely thiazine ring break-down, and the main product is most likely 2,6 -dimethylaniline $[15,18]$; the structure of xylazine and 2,6-dimethylaniline was showed in Figure 1. Due to the qualitative detection ability, chromatography-mass spectrometry has been widely used in biological samples [1922]. The cleanup and target extraction of blood and urine samples have been achieved by different sample preparation methods, such as liquid-liquid extraction (LLE) and solid phase extraction (SPE) [11, 22, 23]. Currently, xylazine detection is usually chromatography or chromatographymass spectrometry methods. The samples are usually animalderived foods such as honey and milk [24, 25]. For human intoxications, the detection target is xylazine in blood [26, 27].

To validate this method and to create a reliable sample preparation method quantitatively to determine the xylazine and its metabolite in body fluids, in this paper, phospholipid removal technology was applied in the detection of xylazine and 2,6-dimethylaniline in body fluid sample. At the same time, the results were verified through a comparison with LLE and SPE.

\section{Material and Methods}

2.1. Chemicals and Reagents. Acetonitrile, methanol, diethyl ether, n-hexane, ethyl acetate, dichloromethane (LC/MS grade, Fisher Scientific, USA), and formic acid (chromatography grade, ROE, USA) were used as received. Water was purified using a Milli-Q water purification system (Millipore, Billerica, MA, USA), Oasis MCX $^{\circledR}(30 \mathrm{mg} / 1 \mathrm{~mL}$; Waters), Cleanert ${ }^{\circledR}$ SLE-AQ $(30 \mathrm{mg} / 1 \mathrm{~mL}$, Bonna-Agela Technologies, China), Ostro ${ }^{\circledR} 96$ wells $(30 \mathrm{mg} / 1 \mathrm{~mL}$; Waters), xylazine (high purity, 98.0\%, J\&K Chemical, China), and 2,6-dimethylaniline (high purity, 98.0\%, J\&K Chemical, China) were used as received, blank blood was supplied by Chongqing city blood center, and blank urine was supplied by healthy laboratory researchers.
2.2. Preparation of Standards and QC Samples. $100 \mathrm{mg} / \mathrm{L}$ stock solutions of xylazine and 2,6-dimethylaniline were prepared by dissolving $1.0 \mathrm{mg}$ chemicals in $10 \mathrm{~mL}$ acetonitrile. After preparation, stock solutions were stored at $-20^{\circ} \mathrm{C}$ until use. Working solution mixtures were prepared by serial dilution of the stock solutions with acetonitrile. Matrixmatched calibration standards and QC samples are done by spiking whole blood and urine with the appropriate volume of the mixed working solution. The concentrations of the matrix-matched calibration standards were $2,5,10,50,100$, 500 , and $2000 \mathrm{ng} / \mathrm{mL}$ and three QC samples at 5, 50, and $500 \mathrm{ng} / \mathrm{mL}$ were prepared for whole blood and urine.

2.3. Instrumentation. LC-MS/MS was performed using a Shimadzu 20A series liquid chromatograph (Shimadzu Corp., Japan) equipped with AB2000 triple quadrupole mass spectrometry (AB Sciex Pte. Ltd., USA). Chromatographic separation was achieved on a Waters Atlantis dC18 $(150 \mathrm{~mm}$ $\times 3.9 \mathrm{~mm}, 5 \mu \mathrm{m})$ LC column; temperature: $40^{\circ} \mathrm{C}$, sample size: $20 \mu \mathrm{L}$, and flow rate: $0.5 \mathrm{~mL} / \mathrm{min}$. Mobile phase $\mathrm{A}$ is an aqueous solution containing $0.1 \%$ formic acid. Mobile phase $\mathrm{B}$ is acetonitrile solution containing $0.1 \%$ formic acid; the mobile phase is programmed to run in the following sequence: $0-1 \mathrm{~min} \mathrm{~A}$ content is $90 \%, 2-3 \mathrm{~min} \mathrm{~A}$ content is decreased from $90 \%$ to $5 \%, 3-6 \mathrm{~min}$ A content is 5\%, 6-7 min A content is increased from $5 \%$ back to $90 \%$, and $7-8$ min A content is $90 \%$.

The electrospray ionization (ESI) conditions for analyte were as follows: nitrogen was used as nebulizing gas (GS 1, 65 psi), turbo spray gas (GS 2, 65 psi), and curtain gas (20 psi). The collision-activated dissociation (CAD) was set to medium level, while the ion spray voltage was at $4000 \mathrm{~V}$. The dwell time was set at 0.1 seconds, and the Mass Spectrum scanned in positive ion mode.

Simultaneous detection of all glycerophosphocholines (GPCho's) using Mass Spectrum detection was based on Little et al. [27]. The Mass Spectrum parameters for AB2000 triple quadrupole mass spectrometry have been optimized by employing a declustering potential, entrance potential, collision energy, and collision cell exit potential of 180, 10, 7 , and $5 \mathrm{~V}$, respectively. The mass transition of $\mathrm{m} / z 184.2 \rightarrow$ 184.2 was monitored in the positive ion electrospray mode.

\subsection{Sample Preparation}

2.4.1. SPE. Solid phase extraction was described in the previous publications $[11,28]$. A $100 \mu \mathrm{L}$ sample was mixed with $200 \mu \mathrm{L}$ of $2 \%$ formic acid and then was vortexed for $10 \mathrm{~s}$ and subjected to SPE. The sample mixture was extracted by Oasis MCX SPE cartridge. The cartridge was activated subsequently by $1 \mathrm{~mL}$ methanol and $1 \mathrm{~mL} 2 \%$ formic acid acidified water right before use. Cartridges were eluted with $2 \mathrm{~mL}$ of $2 \%$ ammonia in methanol and then dried under a stream of heated nitrogen at $40^{\circ} \mathrm{C}$. Finally, the residues were reconstituted in $100 \mu \mathrm{L}$ initial mobile phase solution. A $20 \mu \mathrm{L}$ solution was injected into the LC-MS/MS system for analysis.

2.4.2. SLE. A $100 \mu \mathrm{L}$ sample was mixed with $200 \mu \mathrm{L}$ deionized water and then was loaded onto a Cleanert SLE-AQ 
cartridge. A minimum positive pressure was applied to facilitate the sample absorption into the cartridge in less than $10 \mathrm{~s}$. After the analytes were allowed to equilibrate with the sorbent for $10 \mathrm{~min}$, the analytes were eluted with $1.5 \mathrm{~mL}$ of extraction solvent each time for three times. The extraction solvent was eluted by applying a slightly positive pressure from the top and then evaporated to dryness under a stream of heated nitrogen at $40^{\circ} \mathrm{C}$. Finally, the residues were reconstituted in $100 \mu \mathrm{L}$ initial mobile phase solution. A $20 \mu \mathrm{L}$ solution was injected into the LC-MS/MS system for analysis.

2.4.3. Protein and Phospholipid Removal Cartridge. A volume of $300 \mu \mathrm{L}$ of acetonitrile was transferred to an Ostro 96-well cartridge and then $100 \mu \mathrm{L}$ plasma was added to the cartridge. The Ostro 96-well cartridge was then manually closed and vortexed. After short moderate mixing, the cartridge was applied to positive pressure and the filtrate was collected into a plastic tube ( $2 \mathrm{~mL}$ Axygen). At this point, the filtrate was ready for immediate LC-MS/MS analysis, but to make this method comparable with the other methods with higher sensitivity, it was concentrated by drying under a nitrogen stream at $40^{\circ} \mathrm{C}$ and reconstitution in $100 \mu \mathrm{L}$ of initial mobile phase solution. A $20 \mu \mathrm{L}$ solution was injected into the LCMS/MS system for analysis.

2.5. Method Validation. Matrix-matched calibration curves were constructed by plotting the peak area $(y)$ versus the nominal concentration of the calibration standards $(x)$. The linearity of calibration curves was evaluated by fitting calibration curves by weighted least-squares linear regression with a weighting factor of $1 / x$. Selectivity was investigated by averaging results from five blank samples. The limit of detection (LOD) was set at the lowest concentration yielding at least three signal-to-noise $(S / N)$ ratios with an acceptable chromatographic peak shape. The limit of quantification (LOQ) was the lowest concentration with an $S / N$ ratio greater than 10.

The recovery and matrix effect were evaluated by repeating 6 analyses at $3 \mathrm{QC}$ samples $(5,50$, and $500 \mathrm{ng} / \mathrm{mL})$. The recovery was calculated by dividing the average peak area of the analyte spiked before extraction by the average peak area of the analyte spiked after extraction. The matrix effect was estimated by dividing the average peak area of the analyte spiked after extraction by average peak area derived from the neat standards.

Inaccuracy was determined as the percentage deviation of the average of the results from the corresponding nominal value. Imprecision was expressed as the percent relative standard deviation (\% RSD). Intraday precision assays were determined by analyzing QC samples $(5,50$, and $500 \mathrm{ng} / \mathrm{mL})$ on the same day $(n=6)$.

\section{Results and Discussion}

3.1. Optimization of SPE Method. In current studies, three different extraction methods, SPE, SLE, and protein and phospholipid removal, were chosen for comparison. SPE with Oasis MCX polymeric sorbent cartridge, SLE with Cleanert SLE-AQ cartridge, and protein and phospholipid removal

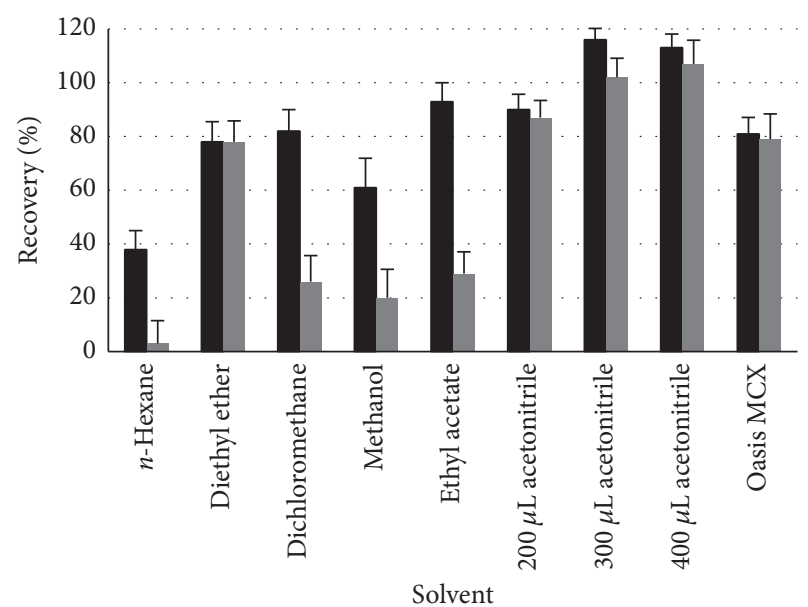

$$
\begin{aligned}
& \text { Xylazine } \\
& \text { DMA }
\end{aligned}
$$

FIGURE 2: Effect of different SPE cartridges on the recoveries of xylazine and DMA.

cartridge with Ostro 96-well cartridge were investigated during the method development. The extraction efficiencies of these three different extraction methods were evaluated by extracting human blood with $100 \mathrm{ng} / \mathrm{mL}$ target addition. As shown in Figure 2, the extraction efficiencies for xylazine with SLE were improved significantly when using ethyl acetate as an eluting solution; the recovery increased from $20 \%$ to $95 \%$. However, all five eluting solutions used were not able to extract 2,6-dimethylaniline effectively; the recovery was only from $7 \%$ to $75 \%$. The extraction efficiencies for xylazine and 2,6-dimethylaniline with SPE according to the literature [17] were $81 \%$ and $75 \%$. In contrast, the extraction efficiencies for xylazine and 2,6-dimethylaniline with Ostro 96-well cartridge are $106 \%$ and $103 \%$, respectively. It is obvious to draw the conclusion that Ostro 96-well cartridge has the highest extraction efficiency based on the above comparison. Therefore, Ostro 96-well cartridge was employed to treat the sample. Ostro 96-well protein and phospholipid removal cartridge facilitated protein precipitation technology for the targeted removal of phospholipids from plasma and serum samples, which was fast, simple, and less time and sample consuming and could significantly reduce the matrix effect. According to the results obtained by Bruce et al. [29], acetonitrile has better protein removal efficiency than methanol in blood samples. The addition of $0.1 \%$ formic acid to acetonitrile is also beneficial for the protein removal. The parameters evaluated in method development need to be optimized for the volume ratio of acetonitrile to blood. Four different ratios of organic solvent to blood were tested: $1: 1,2: 1,3: 1$, and $4: 1(\mathrm{v} / \mathrm{v}) .100 \mu \mathrm{L}$ of blood was used as a blood sample. When the acetonitrile to blood ratio was 1:1, the sample solution is thickened, but there is no protein precipitation. When the ratio was increased to $2: 1$, the protein recoveries were $87 \%$ and $81 \%$ for xylazine and 2,6-dimethylaniline, respectively, suggesting the proteins were not precipitated completely. When the acetonitrile to blood ratio was further increased 


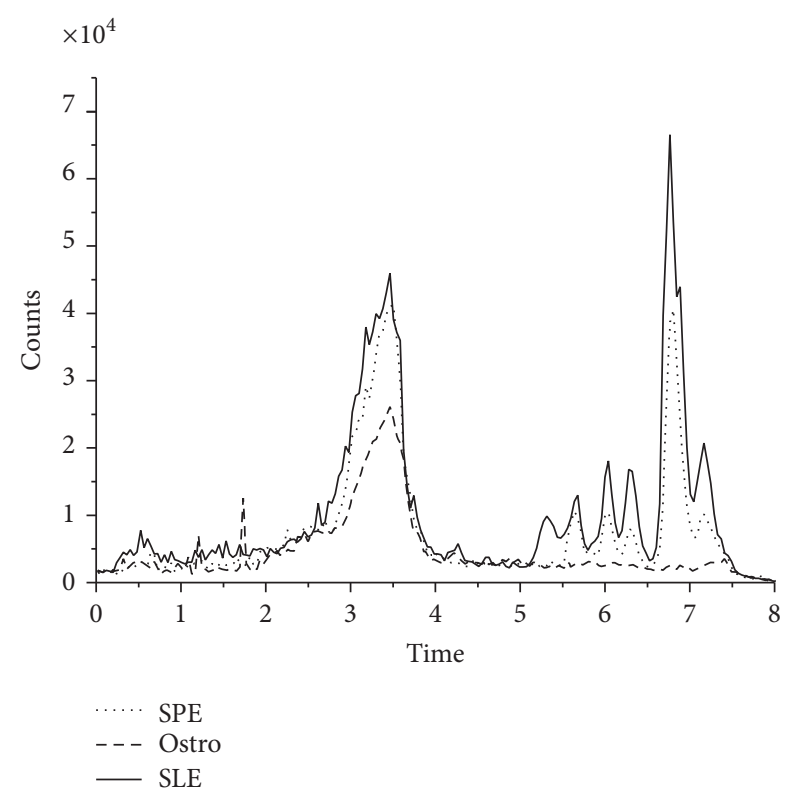

FIGURE 3: GPCho's profiles of three different extraction methods.

to $3: 1$ or $4: 1$, the recoveries for both xylazine and 2,6dimethylaniline were close to $100 \%$. Taking the sensitivity into account, the dilution factor of samples should be as little as possible. Thus, a ratio of $3: 1$ was used.

Glycerophosphocholines (GPCho's) are known to cause liquid chromatography-mass spectrometry/mass spectrometry (LC-MS/MS) matrix ionization effects during the analysis of blood samples. To evaluate the phospholipid removal performance of three methods, in source collision, induced dissociation [30] was employed to monitor the major GPCho's causing matrix effect. Blood samples pretreated by the proposed three methods and their 184.2-184.2 ion couples were monitored by MRM method under the same LC condition. The resultant profile is shown in Figure 3. It is apparent that the phospholipid profiles after different pretreatments differ significantly. As indicated in retention time 2-4 min, phospholipids removal by Ostro cartridge is more efficient than the other two methods, although phospholipids are not completely removed. Also, there are no phospholipid peaks in the mass spectra in the 6.5-7.5 min range of blood sample pretreated by Ostro cartridge method. In contrast, blood samples pretreated by SPE and SLE methods show apparent phospholipid peaks. Thus, it can be concluded that the phospholipid removal efficiency is in the following order: Ostro cartridge > SPE $>$ SLE. Ostro cartridge has the best phospholipid removal and lowest matrix effect, which significantly enhances the target recovery and accuracy. Also, it can provide other potential benefits such as longer LC column lifetime due to the lowered phospholipid content. Regarding pretreatment time, Ostro cartridge method requires the shortest time among the three since it only needs filtration, while SPE requires multiple step procedures to activate and rinse the extraction column and SLE requires a waiting time of 5-10 minutes to reach equilibrium. In other words, Ostro cartridge method is the best among the three for high-throughput sample processing.
3.2. Optimization of Chromatographic Separation. Mobile phase composition and addition would affect the targets separation and ionization efficiencies. Two organic solvents, methanol and acetonitrile, as mobile phases were investigated for the effect of mobile phases on the retention time and peak shapes. When methanol was used as mobile phase, the peak showed an apparent tail and its base had been widening. It had longer retention time too. In contrast, the peak shape improved, and retention time is shorter when acetonitrile was used as mobile phase, at the expense of baseline noise. The amine groups on xylazine and 2,6-dimethylaniline could facilitate ionization under acidic conditions. $0.05 \%, 0.10 \%$, and $0.15 \%$ formic acid were added into mobile phase to study the sensitivity of target compounds at various acidities. The results (not shown) suggested the highest sensitivity at $0.10 \%$ formic acid. There was no significant improvement in sensitivity when increasing formic acid concentration from $0.10 \%$ to $0.15 \%$. The addition of formic acid into acetonitrile mobile phase greatly improved the sensitivity of both xylazine and 2,6-dimethylaniline, which was more than enough to offset the baseline noise. Thus, the mobile phase composition was selected to be $0.10 \%$ formic acid in acetonitrile and water. Such mobile phase, with gradient elution, could be helpful to improve the peak shape and enhance ionization efficiencies.

3.3. Optimization of MS Detection. The amine functional groups on both target compounds could be easily protonated at lower $\mathrm{pH}$ environments, making the two target compounds suitable to be analyzed under positive ion mode. 2,6Dimethylaniline and xylazine protonated molecules $[\mathrm{M}+\mathrm{H}]+$ were observed in the full spectra MS scan under positive mode at $m / z 122$ and $m / z 221$, respectively, when tuned with a single standard solution. After the affirmation of precursor ions, more than two products should be chosen when using LC-MS analysis.

Then MS/MS scan was performed to each precursor ion; the major fragments of 2,6-dimethylaniline were at $\mathrm{m} / z$ 105 and $m / z$ 77, which were selected to construct MRM transitions with precursor ion at $m / z 122$ (Figure 4(a)). Five major fragments of xylazine were observed at $\mathrm{m} / z 164, \mathrm{~m} / z$ $147, m / z 121, m / z 105$, and $m / z 89$ (Figure 4(b)) [25]. Last but not least, MRM transitions with $m / z 164$ and $m / z 89$ for $m / z$ 211 were selected to monitor in the determination of xylazine. The addition of formic acid into mobile phase could enhance ionization efficiencies, target responses, and sensitivities. The optimized parameters, including DP, CE, and CXP, were listed in Table 1.

3.4. Method Validation. In the MRM mode, the high selectivity for the determination of xylazine and 2,6-dimethylaniline in blood and urine samples was found. The typical MS/MS spectra of spiked blood and urine samples and the blank blood and urine samples are depicted in Figure 5. No additional peaks due to endogenous substances that could have interfered with the detection of xylazine and 2,6dimethylaniline were observed. The LODs were 0.3 and $0.2 \mathrm{ng} / \mathrm{mL}$ for xylazine and 2,6-dimethylaniline in blood and urine. The LOQs were 1.0 and $0.6 \mathrm{ng} / \mathrm{mL}$ for xylazine and 2,6-dimethylaniline in blood and urine in Table 2. Standard 
TABLE 1: MS parameters of xylazine and DMA.

\begin{tabular}{lcccrr}
\hline Compound & MW & Precursor/production & DP $(\mathrm{eV})$ & CE $(\mathrm{V})$ & CXP \\
\hline Xylazine & 220 & $221 / 164,221 / 89^{*}$ & $40 / 40$ & $35 / 34$ & $8 / 5$ \\
DMA & 121 & $122 / 105^{*}, 122 / 77$ & $30 / 30$ & $23 / 31$ & $6 / 6$ \\
\hline
\end{tabular}

${ }^{*}$ The quantification production.

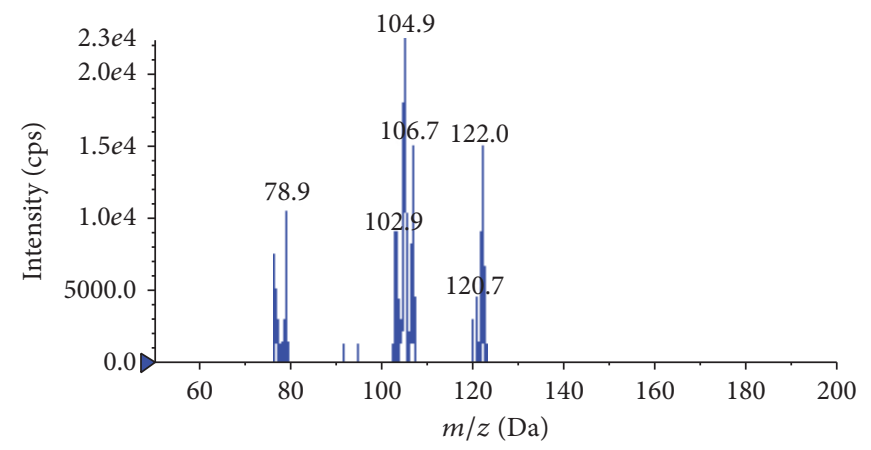

(a)

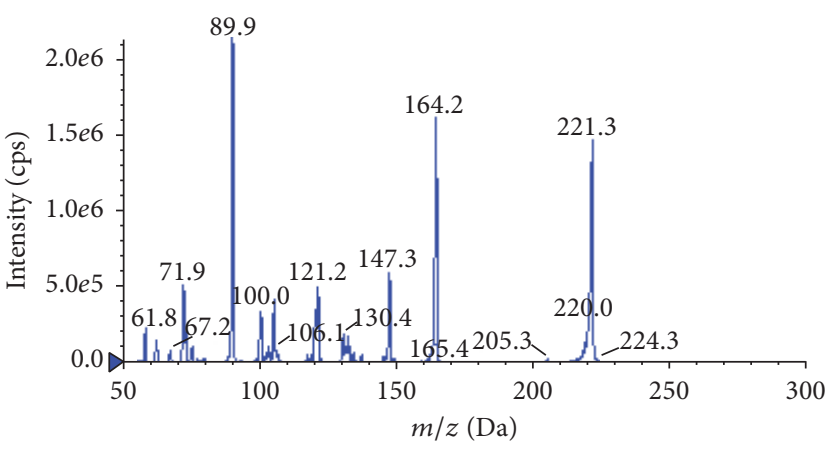

(b)

FIGURE 4: The MS scan spectrum of DMA (a) and xylazine (b).
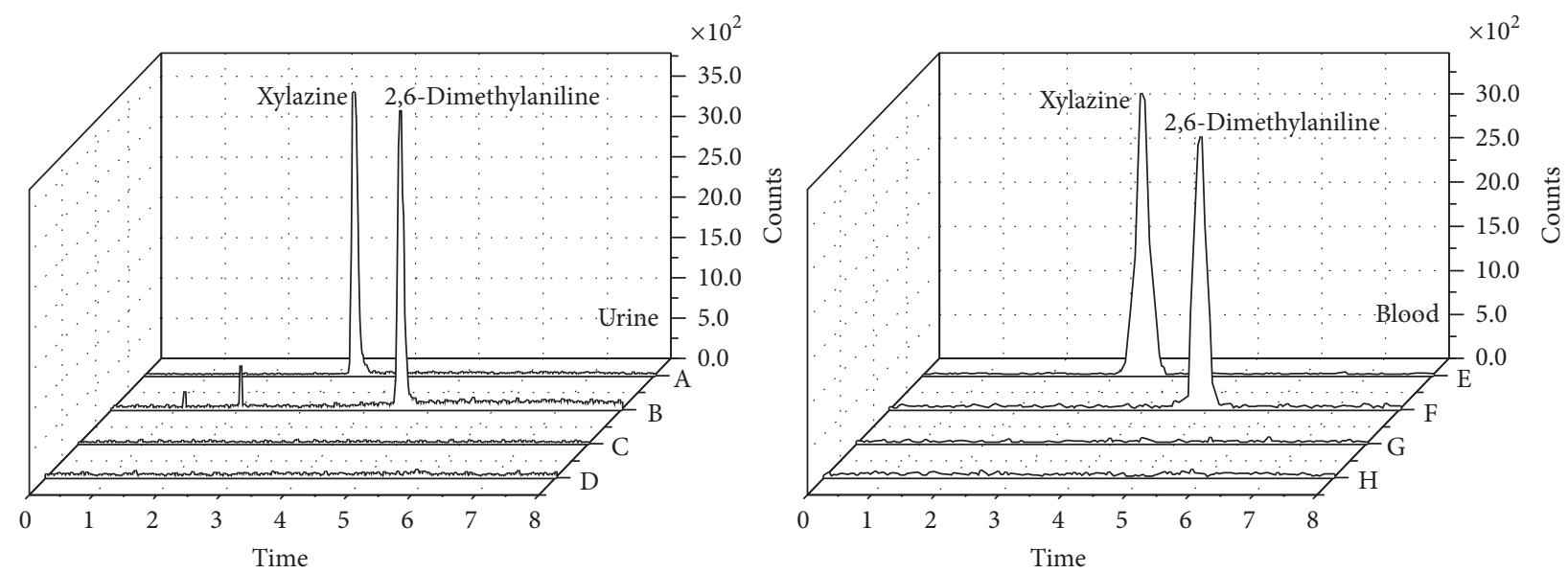

FIGURE 5: Representative MRM chromatograms: (A) blank urine spiked with xylazine (100 ng/mL); (B) blank urine spiked with DMA $(100 \mathrm{ng} / \mathrm{mL}) ;(\mathrm{C})$ blank urine for xylazine; (D) blank urine for 2,6-dimethylaniline; (E) blank blood spiked with xylazine (100 ng/mL); (F) blank blood spiked with DMA (100 ng/mL); (G) blank blood for xylazine; (H) blank urine blood 2,6-dimethylaniline.

calibration curves were linear over the curve range between 2 and $2000 \mathrm{ng} / \mathrm{mL}$ in blood and urine for both xylazine and 2,6-dimethylaniline; the linear regression equations were in Table 2.

Recovery precision and matrix effect (ME) were repeating analysis for each QC sample 6 times. The QC samples were solutions containing 5,50 , and $500 \mathrm{ng} / \mathrm{mL}$ target compounds. The results are summarized in Table 2. The extraction recovery rates were consistent and greater than $93.8 \%$, which indicated that protein and phospholipid removal cartridge was optimal for xylazine and 2,6-dimethylaniline. The precision, assessed by the relative standard deviation (RSD) for the replicate analysis, was typically less than $9 \%$.

Since the selectivity of the HPLC-MS/MS method could be affected by matrix effects by the sample matrix and interferences from compound metabolites, matrix effects are investigated to develop a more specific and reliable method. The matrix effect ranged from 101.1 to $108.2 \%$ for both analytes. This result suggested that ion suppression or enhancement from the blood and urine matrix was negligible under the current treatment method. With a low matrix effect and consistent and reproducible recovery, this assay has proved to be reliable for bioanalysis.

3.5. Application to Actual Samples. A 36-year-old woman ingested a certain amount of xylazine after a quarrel with her husband and became unconscious. She was immediately sent to the hospital when her family discovered and was found dead at the time of arriving at hospital. A heart blood sample was collected from the subject for toxicological analysis; xylazine and 2,6-dimethylaniline were confirmed and quantified at $267 \mathrm{ng} / \mathrm{mL}$ and $451 \mathrm{ng} / \mathrm{mL}$ in the whole blood sample. 


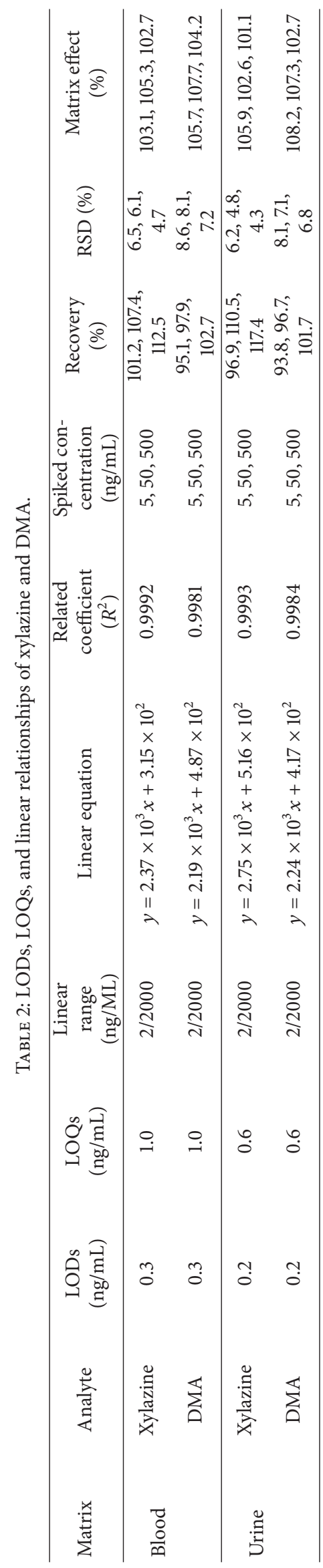




\section{Conclusions}

In this study, an HPLC-MS/MS method for the quantification of xylazine and 2,6-dimethylaniline in blood and urine samples was developed and validated. A rapid, sensitive, specific, selective, and reproducible assay was developed. Also, the assay required small sample size and simple protein and phospholipid removal cartridge extraction without additional procedures. The sensitive method was successfully applied for the detection of xylazine and 2,6-dimethylaniline in a blood sample for clinical diagnosis and forensic toxicology.

\section{Competing Interests}

The authors declare that there is no conflict of interests regarding the publication of this paper.

\section{Acknowledgments}

This work was supported by Open Foundation of Chongqing Technology and Business University.

\section{References}

[1] D. Zhu, H. Cheng, J. Li et al., "Enhanced water-solubility and antibacterial activity of novel chitosan derivatives modified with quaternary phosphonium salt," Materials Science and Engineering $C$, vol. 61, pp. 79-84, 2016.

[2] Y. Chen, J. Li, Q. Li et al., "Enhanced water-solubility, antibacterial activity and biocompatibility upon introducing sulfobetaine and quaternary ammonium to chitosan," Carbohydrate Polymers, vol. 143, pp. 246-253, 2016.

[3] Z. Bin, Z. Wenwen, and Z. Yiwen, "Study on the application of initiator producing graft modified starch size mixture," Cotton Textile Technology, vol. 5, pp. 19-22, 2008.

[4] Q. Xu, J. Wei, J. Wang et al., "Facile synthesis of copper doped carbon dots and their application as a 'turn-off' fluorescent probe in the detection of $\mathrm{Fe}^{3+}$ ions," RSC Advances, vol. 6, no. 34, pp. 28745-28750, 2016.

[5] W. Zhang, N. R. Vinueza, P. Datta, and S. Michielsen, "Functional dye as a comonomer in a water-soluble polymer," Journal of Polymer Science, Part A: Polymer Chemistry, vol. 53, no. 13, pp. 1594-1599, 2015.

[6] L. Yao, M. Jiang, D. Zhou et al., "Fabrication and characterization of microstrip array antennas integrated in the three dimensional orthogonal woven composite," Composites Part B: Engineering, vol. 42, no. 4, pp. 885-890, 2011.

[7] Y. Kuang, A. Yang, H. Luan et al., "Effects of structural parameters of textile substrates on the novel textile conformal halfwave dipole antenna," Journal of Industrial Textiles, 2016.

[8] L. Yao and Y. Qiu, "Design and fabrication of microstrip antennas integrated in three dimensional orthogonal woven composites," Composites Science and Technology, vol. 69, no. 7-8, pp. 1004-1008, 2009.

[9] D. Zhou, L. Yao, L. Fei, Z. Da, and M. Jiang, "Tensile and shear properties of three dimensional orthogonal woven basalt/kevlar hybrid composites," Fiber Composites, vol. 1, article 017, 2010.

[10] X. Gong, "Controlling surface properties of polyelectrolyte multilayers by assembly pH," Physical Chemistry Chemical Physics, vol. 15, no. 25, pp. 10459-10465, 2013.
[11] X. Gao, H. Guo, Y. Du, and C. Gu, "Simultaneous determination of xylazine and 2,6-xylidine in blood and urine by auto solidphase extraction and ultra high performance liquid chromatography coupled with quadrupole-time of flight mass spectrometry," Journal of Analytical Toxicology, vol. 39, no. 6, pp. 444-450, 2015.

[12] J. Wang, P. Jiang, L. Gao et al., "Unique self-assembly properties of a bridge-shaped protein dimer with quantum dots," Journal of Nanoparticle Research, vol. 15, no. 9, article 1914, 2013.

[13] D. Ling, L. Gao, J. Wang et al., "A general strategy for sitedirected enzyme immobilization by using $\mathrm{NiO}$ nanoparticle decorated mesoporous silica," Chemistry, vol. 20, no. 26, pp. 7916-7921, 2014.

[14] T. Ribeiro, T. J. V. Prazeres, M. Moffitt, and J. P. S. Farinha, "Enhanced photoluminescence from micellar assemblies of cadmium sulfide quantum dots and gold nanoparticles," The Journal of Physical Chemistry C, vol. 117, no. 6, pp. 3122-3133, 2013.

[15] L. I. Velez, G. Shepherd, L. D. Mills, and W. Rivera, "Systemic toxicity after an ocular exposure to xylazine hydrochloride," Journal of Emergency Medicine, vol. 30, no. 4, pp. 407-410, 2006.

[16] U. Hoffmann, C. M. Meister, K. Golle, and M. Zschiesche, "Severe intoxication with the veterinary tranquilizer xylazine in humans," Journal of Analytical Toxicology, vol. 25, no. 4, pp. 245249, 2001.

[17] K. A. Moore, M. G. Ripple, S. Sakinedzad, B. Levine, and D. R. Fowler, "Tissue distribution of xylazine in a suicide by hanging," Journal of Analytical Toxicology, vol. 27, no. 2, pp. 110-112, 2003.

[18] R. Garcia-Villar, P. L. Toutain, M. Alvinerie, and Y. Ruckebusch, "The pharmacokinetics of xylazine hydrochloride: an interspecific study," Journal of Veterinary Pharmacology and Therapeutics, vol. 4, no. 2, pp. 87-92, 1981.

[19] H. Guo, P. Zhang, J. Wang, and J. Zheng, "Determination of amitraz and its metabolites in whole blood using solid-phase extraction and liquid chromatography-tandem mass spectrometry," Journal of Chromatography B: Analytical Technologies in the Biomedical and Life Sciences, vol. 951-952, no. 1, pp. 89-95, 2014.

[20] Z. Yu, T. Kabashima, C. Tang et al., "Selective and facile assay of human immunodeficiency virus protease activity by a novel fluorogenic reaction," Analytical Biochemistry, vol. 397, no. 2, pp. 197-201, 2010.

[21] I. J. Smallman, F. Wang, T. C. Steimle, M. R. Tarbutt, and E. A. Hinds, "Radiative branching ratios for excited states of $174 \mathrm{YbF}$ : application to laser cooling," Journal of Molecular Spectroscopy, vol. 300, pp. 3-6, 2014.

[22] Q. Han, Z. Wang, J. Xia, X. Zhang, H. Wang, and M. Ding, "Application of graphene for the SPE clean-up of organophosphorus pesticides residues from apple juices," Journal of Separation Science, vol. 37, no. 1-2, pp. 99-105, 2014.

[23] S. Kumar Pandey and K.-H. Kim, "Evaluation of the solid-phase extraction (SPE) cartridge method in combination with thermal desorption-gas chromatography-mass spectrometry (TD-GCMS) for the analysis of different VOCs in liquid matrices in varying $\mathrm{pH}$ conditions," Journal of Separation Science, vol. 35, no. 15, pp. 1914-1921, 2012.

[24] X. Zheng, X. Mi, S. Li, and G. Chen, "Determination of Xylazine and 2,6-Xylidine in animal tissues by liquid chromatographytandem mass spectrometry," Journal of Food Science, vol. 78, no. 6, pp. T955-T959, 2013.

[25] P. Delahaut, P.-Y. Brasseur, and M. Dubois, "Multiresidue method for the detection of tranquillisers, xylazine, and a 
$\beta$-blocker in animal production by liquid chromatographytandem mass spectrometry," Journal of Chromatography A, vol. 1054, no. 1-2, pp. 373-378, 2004.

[26] K. Ruiz-Colón, C. Chavez-Arias, J. E. Díaz-Alcalá, and M. A. Martínez, "Xylazine intoxication in humans and its importance as an emerging adulterant in abused drugs: a comprehensive review of the literature," Forensic Science International, vol. 240, pp. 1-8, 2014.

[27] J. L. Little, M. F. Wempe, and C. M. Buchanan, "Liquid chromatography-mass spectrometry/mass spectrometry method development for drug metabolism studies: examining lipid matrix ionization effects in plasma," Journal of Chromatography B, vol. 833, no. 2, pp. 219-230, 2006.

[28] X. Gao, H. Guo, W. Zhang, and C. Gu, "Simultaneous determination of amitraz and its metabolites in blood by support liquid extraction using UPHLC-QTOF," Journal of Analytical Toxicology, vol. 40, no. 6, pp. 437-444, 2016.

[29] S. J. Bruce, I. Tavazzi, V. Parisod, S. Rezzi, S. Kochhar, and P. A. Guy, "Investigation of human blood plasma sample preparation for performing metabolomics using ultrahigh performance liquid chromatography/mass spectrometry," Analytical Chemistry, vol. 81, no. 9, pp. 3285-3296, 2009.

[30] G. M. J. Meyer and H. H. Maurer, "Qualitative metabolism assessment and toxicological detection of xylazine, a veterinary tranquilizer and drug of abuse, in rat and human urine using GC-MS, LC-MS n, and LC-HR-MS n," Analytical and Bioanalytical Chemistry, vol. 405, no. 30, pp. 9779-9789, 2013. 

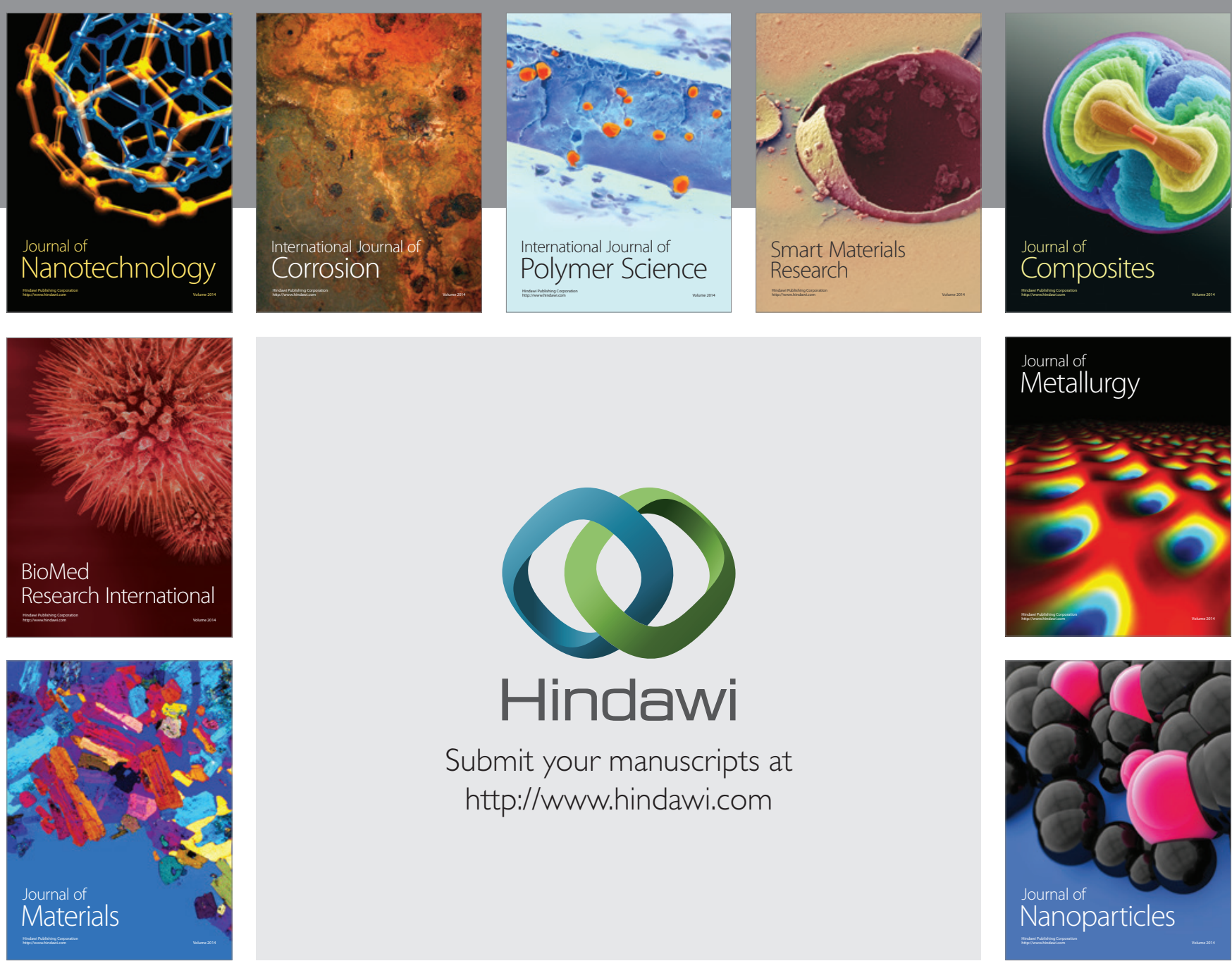

\section{Hindawi}

Submit your manuscripts at

http://www.hindawi.com

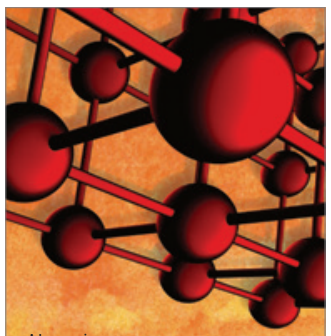

Materials Science and Engineering
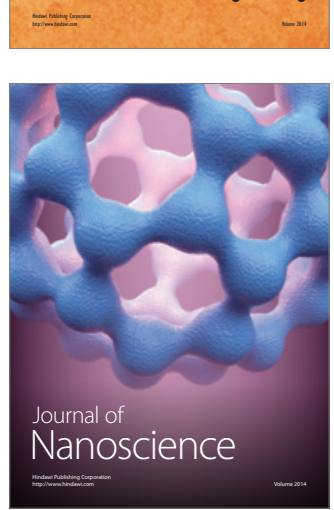
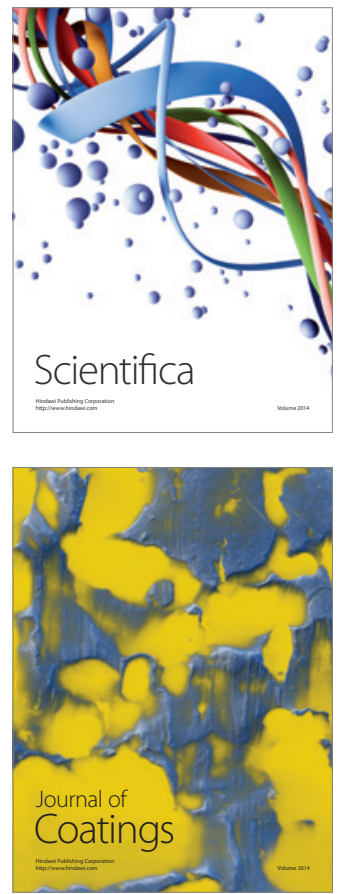
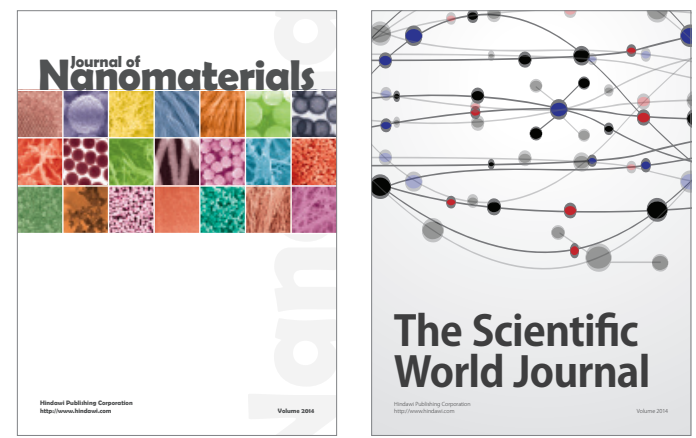

The Scientific World Journal
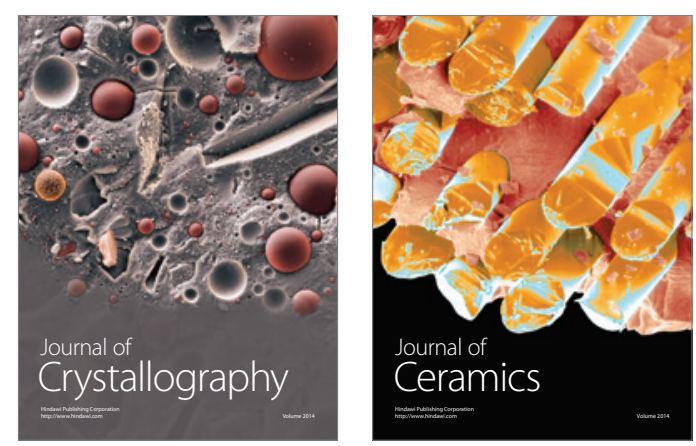
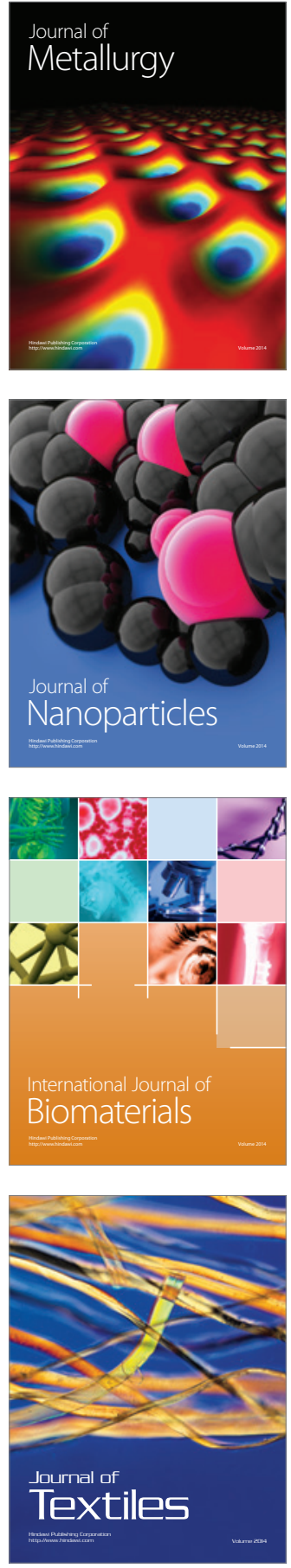